\title{
Techniques and Applications in Instructional L2 Learning
}

\author{
Hulin Ren ${ }^{1 \& 2}$ \\ ${ }^{1}$ Foreign Language Division, Beijing Union University, Beijing, China \\ ${ }^{2}$ Foreign Language Department, North China Electric Power University, Beijing, China \\ Correspondence: Hulin Ren, Foreign Language Division, Beijing Union University, Beijing, China. E-mail: \\ hulinr@aliyun.com; hulinr@hotmail.com
}

Received: August 2, 2017 Accepted: August 25, 2017 Online Published: September 25, 2017

doi:10.5539/ijel.v7n6p198 URL: http://doi.org/10.5539/ijel.v7n6p198

\begin{abstract}
In this paper, a survey of certain techniques applied in the study of second language (L2) learning is presented. In this context, four types of techniques with reference to two critical questions in investigating instructional learning process of L2 sentences are described and commented: (1) how do individual L2 learners' differences process sentences in real time?; (2) what is the decisive factor in L2 learners' sentence processing? The aim is to show how comprehensive techniques that capture real-time sentence processing of L2 learners with instruction can be applied to elucidate such questions, with the purpose to suggest best suited techniques to particular research topics in exploring learners' nature of instructional L2 sentence processing.
\end{abstract}

Keywords: L2 learners, sentence processing, on-line techniques

\section{Introduction}

In recent years, a number of published studies in L2 learning using different techniques tend to be increasing. Among which, traditional techniques such as grammaticality judgments (GJs) are popular in the study of L2 learners' final achievements. Meanwhile, there is an increasing trend to use online or psycholinguistic techniques to address central questions in the field of L2 learning at instructional setting, such as how do learners process L2 sentences in real time under explicit grammatical instruction? Since processing L2 sentences involves both the accumulation of instructional L2 knowledge and the ability to put that knowledge to use in real-time processing (Roberts, 2012). Within the study of L2 sentence processing of learners, one debatable issue concerns the nature of learners' L2 sentence processing, i.e., whether L2 learners' differences in sentence processing are rule-based or statistics-based processing.

The rule-based approach to L2 sentence processing assumes that sentence processing is a rule-governed set of operations constrained by working memory capacity. Individual differences in L2 sentence comprehension are related to L2 learners' individual differences in the size of working memory (e.g., Felser et al., 2003). However, the statistics-based approach argues that sentence processing is based on statistical regularities. Thus, individual differences in L2 sentence comprehension are explained in terms of learners' differences in L2 exposure or frequency (e.g., Gregg, 2003). To have a clear picture of L2 sentence processing, a number of studies (e.g. Mandell, 1999; White, 1989) have investigated the rule-based and/or the statistics-based sentence processing with L2 learners, in so doing, techniques are applied in these studies with the purpose to obtain reliable data exploring the determining factor in L2 learners' sentence processing.

In section 2 and 3, a summary of different types of techniques available in L2 sentence processing is made, and described with a brief comment of studies that have made use of these techniques. Comments and suggestions can speak to current issues in L2 sentence processing. Conclusions (i.e., section 4) are drawn with regard to further investigation of L2 sentence processing by means of combined techniques.

\section{Grammaticality Judgments (GJs)}

Many studies in the field of L2 sentence processing have used the technique of GJs, which is generally taken to measure L2 learners' linguistic knowledge underling their language performance (Mandell, 1999). According to Schütze (1996), in order to observe how learners' processing L2 sentences and the role of linguistic knowledge in L2 sentence processing, an excellent and reliable source of information about learners' grammar can be observed from GJs. For Schütze, the use of GJs in the study of sentence processing lies in the following four 
merits.

Firstly, by the use of eliciting judgments, reactions to sentence types occurring only very rarely in spontaneous speech or recorded corpora can be examined, which are beneficial for observing L2 learners' syntactic knowledge while speaking. Secondly, using GJs can obtain a form of information that scarcely exists within normal language use, such as negative information in the form of strings that are not part of the language. Thirdly, on the occasion that one is merely observing speech, it is hard to distinguish reliably slips and unfinished utterances. Under this circumstance, grammatical production can provide the related data in this regard. Lastly, by using GJs, one is able to minimize the extent to which the communicative and representational functions of language skill obscuring our insight into its related mental nature (1996, p. 16).

White $(1989,2003)$ further assumed that GJs used in L2 study could gain an insight into learners' linguistic competence. In other words, using GJs may provide an adequate measure of what L2 learners know, and the related function of this linguistic competence in sentence processing. Mandell (1999) confirmed that GJ data are reliable measures of linguistic knowledge in L2 learners, by comparing the GJ test data with dehydrated sentence test data (i.e., an assessment tool commonly used in the L2 classroom) from adult L2 learners of Spanish about verb movement, Mandell found that the two different tests are consistent for adult L2 learners of Spanish from the second, fourth and sixth semester at school setting. Unfortunately, Mandell did not mention the role of the instructional knowledge in L2 sentence processing with these learners, nor show any evidence of the determining factor in the study of sentence processing. Hopp \& Arriaga (2016) employed grammatical and ungrammatical case marking for Spanish sentence types in GJs. In their study, GJs were taken in the form of the accurate percentage of acceptance. The results show that non-native learners are only sensitive to violations of structural case marking in processing of case in Spanish with ditransitive verbs, but still not discussing the deciding factor in processing structuring case marking.

One practical advantage of GJs is its relative ease to be used in studies, as it does not require any special apparatus or software, and hence it is a commonly used technique when investigating instructional syntactic construction in sentence processing with L2 learners in particular. In addition, many linguists generally acknowledge that GJs are reliable evidence about the nature of learners' underlying linguistic representations at formal schooling setting, therefore, which might be unlikely to be dismissed as "mere performance" (cf. Wasow $\&$ Arnold, 2005). In consideration of the advantages described above, GJ technique is taken as one traditional technique to investigate L2 learning outcome in comprehension or production.

In spite of the strengths of GJs described above, there are also criticisms. One of the criticisms is that the data elicited from GJs may be inconclusive about why a learner rejects or accepts a sentence in the course of learning processing (Gass, 2001). Furthermore, Juffs (2006) took that in making GJ "learners may bring irrelevant metalinguistic knowledge to bear on subtle linguistic knowledge that is the focus of a study". In addition, data from GJs in sentence processing can only provide indirect evidence about the real-time learning process at sentence level, and consequently lack direct relevant evidence to specific issues, for example, the issue which consumes reading times or the time taken to respond to a sentence in the real-time processing with L2 learners. Moreover, judging the grammaticality of a sentence is influenced by a variety of factors: plausibility, complexity, fatigue, mode of presentation, and so on. One is difficult to clarify which factor functions dominantly in L2 learners' judgment, and therefore, data elicited from this technique should be interpreted with caution (Dabrowska \& Street, 2006). This clearly indicates that this technique precisely lacks defining the nature of L2 learners' differences in sentence processing. The great challenge of GJs may be it is unlikely to provide information on how exactly instructional grammatical information is used in real time sentence processing and thus not being able to reflect the real function of instructional knowledge in sentence comprehension (Sabourin $\&$ Stowe, 2008). This challenge can be taken by using online techniques such as event-related potential techniques described in the following section.

\section{Online Techniques for L2 Sentence Processing}

Compared with the weak points of GJs mentioned above, online techniques may have advantage in exploring the real-time sentence processing with L2 learners, as these techniques can provide speed response to determine real processes of instruction underlying sentence comprehension (Roberts, 2012). Below some of the available techniques in online sentence processing studies with L2 learners are described. Among which, event-related potential (ERP) technique is introduced first, followed by eye-tracking and the self-paced reading techniques.

\subsection{ERP Technique}

ERP technique seems to be ideally used in L2 sentence processing, for its exact information of learners' brain responses to syntactic information, such as the data of syntactic violations by using ERP technique can be 
supplementary to the response of its instructional grammatical counterparts, since ERP can provide scalp-recorded changes in electrical activity that occur in response to a sensatory, cognitive, or more brain-related events with language learners (Rugg \& Coles, 1995). The scalp-recorded ERP is sensitive to particular aspects of sentence processing, reflected by small scalp-recorded voltage changes in a continuously measured electroencephalogram (McKinnon \& Osterhout, 1996; Mueller, 2005), which are precisely time-locked to an external event assumed to be closely related to the processing mechanism in L2 learners' studies, such as instructional syntactic knowledge in L2 learners' sentence processing.

ERP has several components, among which, the most extensively studied one directly linked to learners' language processing is the N400, which is taken as an index of semantic incongruity. Generally speaking, N400 is a centro-parietal negativity around $400 \mathrm{~ms}$, taken closely related to semantic integration processes, which is regarded as a semantic function of 12 learners between the target word and the given context (McKinnon \& Osterhout, 1996). Another component is a centro-parietal positivity peaking around $600 \mathrm{~ms}$, called P600, index of syntactic integration, reanalysis, and repair. Besides, syntax-related negativities occurring prior to the P600 may take place with language learners as well, which often have a left-anterior scalp distribution (Friederici, 2002; Kutas \& VanPetten, 1994).

The strength of ERP lies in its direct reflection of learners' brain activity, as stated by Clifton et al. (1994): "with an electrophysiological marker of some cognitive process in hand, one can infer changes in the underlying cognitive process directly from changes in the ERP component" (19). The ERP components can reflect the summed, simultaneously occurring postsynaptic activity within neocortical pyramidal neurons, which can provide direct evidence of neuron activities in L2 sentence processing with language learners. Topographical features of the ERP, including polarity (positive and negative), amplitude, onset, peak latency, and scalp distribution, should be noted as well, since they can provide a millisecond-by-millisecond record of language learners' brain electrical activity in sentence processing. In addition, ERPs are multidimensional in terms of its varying in polarity, latency, source configurations, which is taken as an ideal tool to examine language learners' different subprocesses during comprehension, mainly beneficial for investigating dominant factors in L2 sentence processing (cf. Rugg \& Coles, 1995).

Despite the strength of accurately reflecting learners' brain electrical activity in L2 sentence processing, the weakness of ERPs is that, making inferences about the timing of cognitive processes based on the ERP data, is to some extent fraught with danger, especially under the condition that the ERP effects are occurring relatively late, it is hard to make accurate inferences of the role of instructional knowledge in L2 sentence processing. Another problem is, as suggested by Osterhout et al. (2004), it is insignificant to employ this technique unless having necessary sensitivity to the process of interest in sentence processing with L2 learners. Below (section 3.2) is a description of another frequently used technique in L2 sentence processing, i.e., eye-tracking technique.

\subsection{Eye-tracking Technique}

Like ERPs, eye-tracking technique can provide continuous measurement during L2 learners' instructional sentence processing. It is quite useful for modeling certain aspects of sentence reading comprehension (Osterhout et al., 2004). The measurement of L2 learners' reading by means of eye-movements provides a multifaceted trace of L2 learners' processing of sentences. There are various different standards of eye-movements. Take the measurement of time in sentence processing as an example: there are first-pass reading times, second-pass reading times and total reading times (i.e., total sum of the former two measures) for any region of interest. Some studies (e.g., Hopp \& Arriaga, 2016) take first-pass reading times as index of first pass syntactic processing, and second-pass reading times are taken as the summed length of all fixations in a sentence region.

Frenck-Mestre $(2005,2005)$ assumed that first time reading can furthermore be broken up into first fixation, namely, the first time the eyes landed in a given region of interest, and gaze duration, that is, all fixations in the region of interest from the first fixation until the eyes exit to the right or to the left. In sum, by using eye-tracking technique, the time of L2 learners' eye-movements is recorded and measured while they read (process) these specific constructions. The recorded processing time usually includes first-pass reading times and second-pass reading times. The former reflects the initial reading of a particular word or phrase and the latter reflects regressions and rereads of the material. The first and second reading times is often associated with initial parsing decisions or re-analysis, which are taken as the valid data reflecting the real time course of instructional L2 sentence processing (Rayner et al., 1989).

In addition to providing the measurement of time, fixations from eye-tracking can also provide other indications of L2 sentence processing under instruction: one can calculate the number of first-pass fixation and re-fixations 
in an instructional region, and determine both length of saccades between fixations and the probability of skipping over an instructional region in a sentence. All these data can be indicative of processing difficulty in instructional sentences, as pointed out by Frenck-Mestre (2005) "the pattern of regression and the probability of re-fixating a region provide valuable information on the eventual difficulty of text processing" (176).

One advantage of eye-tracking is it can provide a multifaceted trace of instructional sentences processing with L2 learners; and the recording can translate both L2 learners' initial and subsequent processing of an instructional sentence. The other advantage is it can provide very useful data to analyze when (e.g., during the first or second pass through a sentence immediately) and where (e.g., at an instructional disambiguating point for example, prior thereto, immediately thereafter, etc.) processing difficulty of sentences occur and how L2 learners keep their eyes on a particular instructional region while in L2 sentence processing.

The disadvantage of eye-tracking technique lies in that eye movements respond similarly to events occurring at different levels of sentence processing. One example is that eye fixations and regressions occur following an anomaly at any sentence level, and thus hard to clarify the exact instructional role in the course of L2 sentence processing. The other is it does not provide any direct information about how sentence processing is instantiated in the brain, and the determining factor of L2 sentence processing in particular. Furthermore, prior to using eye-movements technique, one must ensure all factors are controlled well in order for one to be able to draw any useful conclusions from the data. For instance, the linguistic variables, such as lexical frequency and predictability of the word in the sentence context, should be controlled. Below (section 3.3) is an introduction of the self-paced reading technique, which is also popular in L2 learners' sentence processing.

\subsection{The Self-paced Reading Technique}

The self-paced reading technique is another online technique that requires subjects to read a sentence in a word-by-word or phrase-by-phrase one after another (Marinis, 2003). This technique requires subjects to read each word or phrase as fast as possible, and press a push button to see the next word or phrase after instruction. Comprehension questions are presented following the sentences so as to ensure meaningful online processing of the sentence.

A number of studies that examines sentence processing among L2 learners have begun to adopt self-paced reading technique, often in a mixed combination of measuring reading times and accurate rates in sentence comprehension. For this technique, reading times are regarded as a measure of the relative processing difficulty of a particular sentence or grammatical structure. Longer reading times indicates greater processing difficulties of one given instructional sentences, and the vice versa.

This technique normally entails three possible types of visual presentation: the cumulative presentation, the noncumulative presentation and the centre noncumulative presentation. Sentences appeared on the screen with nonspace characters are replaced by dashes. By pressing the push button, the first word or phrase will be displayed, replacing the corresponding dashes. On the second time of pressing the push button, the next word or phrase is displayed on the screen and this goes on until the end of the sentence. Cumulative and noncumulative presentation are different in that previous words or phrases remain displayed on the screen in the former presentation, whereas in noncumulative presentation the previous words or phrase is disappeared and only one word or phrase is displayed on a screen at a time. In the noncumulative centre presentation, without dashes on the screen, words or phrases are presented in a screen centre.

In recent studies, the self-paced reading technique seems to be widely used in instructional L2 sentence processing studies, as self-paced reading tasks can yield on-line reaction time measures providing a record of L2 learners' performance during sentence comprehension (Gregg, 2003; Papadopoulou, 2005). In most L2 sentence studies, it is popular to use the self-paced reading technique for L2 sentence processing, in so doing, instructional L2 sentences are presented either word-by-word or phrase-by-phrase style. The instructional sentences are divided into several segments. Once a L2 learner reads the segmented sentences on a computer screen, the appearance of the segmented sentences is triggered by pressing the button from the L2 learner. Each time the L2 learner presses the button will be recorded by the computer. The time that the $\mathrm{L} 2$ leaner takes to request the next word or phrase can be measured. By calculating the total amount of times with the leaner can inform L2 sentence processing in real time.

As stated above, the self-paced reading technique can be conducted in cumulative or noncumulative manner. The strength of the noncumulative technique is that L2 learners cannot look back to the words or phrases that had previously been read or instructed. This may encourage L2 learners to keep up to date with their processing (Mitchell \& Green, 1978). Clearly, one main advantage of this technique is that it is a quick and readily available tool, which can provide available information for instructional L2 sentence processing. The disadvantage of this 
technique, according to Frenck-Mestre (2005), is that initial analysis and re-analysis are difficult to be distinguished. This means that it can generally provide a single measure of the total reading time of a given word/segment or sentence, but difficult to precisely define the determining factor in L2 learners' sentence processing with instruction.

\section{Conclusion}

Four types of techniques applied in the study of instructional L2 learning at the level of sentence processing are reviewed in this paper. One common technique of untimed GJs in instructional L2 sentence processing is described. Meanwhile, three online techniques, namely, ERP technique, eye-tracking and self-paced reading techniques, are also discussed. It shows that the described four types of techniques in L2 study at sentence level have both advantages and disadvantages; only one type of technique is hard to give enough evidence illustrating the rule-based or statistics-based account of L2 learners' sentence processing. Therefore, it seems that a complete picture of L2 sentence processing with instruction will need comprehensive techniques, and the mixed application of at least two techniques presented here can provide reliable and convincing data, which may inform the true story of L2 learners' sentence processing under instruction.

\section{Acknowledgments}

This work was supported by Beijing Social Science Key Planning Project Fund (2015BJ0122, Beijing, China).

\section{References}

Clifton, C. J., Frazier, L., \& Rayner, K. (1994). Perspectives on Sentence Processing. Hillsdale, New Jersey Hove: Lawrence Erlbaum Associates, Inc.

Dabrowska, E., \& Street, J. (2006). Individual differences in language attainment: comprehension of passive sentences by native and non-native English speakers. Language Science, 28(16), 604-615. https://doi.org/10.1016/j.langsci.2005.11.014

Felser, C., \& Roberts, L., \& Marinis, T., \& Gross, R. (2003). The processing of ambiguous sentences by first and second language learners of English. Applied Psycholinguistics, 24, 453-489. http://dx.doi.org/10.1007/s11525-007-9116-8

Frenck-Mestre, C. (2005). Ambiguities and anomalies: what can eye movements and event-related potentials reveal about second language sentence processing? In J. F. Kroll \& A. M. B. DeGroot (Eds.), Handbook of Bilingualism:Psycholinguistic Perspectives (pp. 268-281). New York: Oxford University Press.

Frenck-Mestre, C. (2005). Eye-movement recording as a tool for studying syntactic processing in a second language: a review of methodologies and experimental findings. Second Language Research, 21(2), 175-198. https://doi.org/10.1191/0267658305sr257oa

Friederici, A. D. (2002). Towards a neural basis of auditory sentence processing. Trends in Cognitive Sciences, 6 , 78-84. https://doi.org/10.1016/S1364-6613(00)01839-8

Gass, S. (2001). Innovations in second language research methods. Annual Review of Applied Linguistics, 21, 221-232. https://doi.org/10.1017/S0267190501000137

Gregg, K. R. (2003). The state of emergentism in second language acquisition. Second Language Research, 19. 95-128. https://doi.org/10.1191/0267658303sr213oa

Hopp, H., \& Arriaga, M. E. (2016). Structural and inherent case in the non-native processing of Spanish: constraints on inflectional variability. Second Language Research, 32(1), 75-108. https://doi.org/10.1191/0267658303sr213oa

Juffs, A. (2006). Grammar and parsing and a transition theory. Applied Psycholinguistics, 27, 69-91. https://doi.org/10.1017/S0142716406060115

Kutas, M., \& VanPetten, C. K. (1994). Psycholinguistics electrified: event-related brain potential investigations. In M. A. Gernsbacher (Ed.). Handbook of Psycholinguistics, New York: Academic Press. 83-143.

Mandell, P. B. (1999). On the reliability of grammaticality judgment tests in second language acquisition research. Second Language Research, 1, 73-99. https://doi.org/10.1191/026765899667538959

Marinis, T. (2003). Psycholinguistic techniques in second language acquisition research. Second Language Research, 192, 144-161. https://doi.org/10.1191/0267658303sr217ra

McKinnon, R., \& Osterhout, L. (1996). Constraints on movement phenomena in sentence processing: evidence from event-related brain potentials. Language and Cognitive Processes, 11, 495-523. 
http://dx.doi.org/10.1080/016909696387132

Mitchell, D. C., \& Green, D. W. (1978). The effects of context and content on immediate processing in reading. Quarterly Journal of Experimental $\quad$ Psychology, $\quad 30(4), \quad 609-636$. http://dx.doi.org/10.1080/14640747808400689

Mueller, J. L. (2005). Electrophysiological correlates of second language processing. Second Language Research, 21(2), 152-174. https://doi.org/10.1191/0267658305sr256oa

Osterhout, L., McLaughlin, J., Ariji, K., \& Inoue, K. (2004). Sentences in the brain: event-related potentials as real-time reflections of sentence comprehension and language learning. In M. Carreiras \& C. Clifton (Eds.), The On-line Study of Sentence Comprehension: Eyetracking, ERP, and Beyond (Chapter 14, pp. 271-308). Philadelphia: Psychology Press.

Papadopoulou, D. (2005). Reading-time studies of second language ambiguity resolution. Second Language Research, 21, 98-120. https://doi.org/10.1191/0267658305sr254oa

Rayner, K., Sereno, S. C., Morris, R. K., Schmauder, A. R., \& Clifton, C. J. (1989). Eye movements and on-line language comprehension processes. Language and Cognitive Processes, 4, 21-49. http://dx.doi.org/10.1080/01690968908406362

Roberts, L. (2012). Review article: psycholinguistic techniques and resources in second language acquisition research. Second Language Research, 28(1), 113-127. https://doi.org/10.1177/0267658311418416

Rugg, M. D., \& Coles M. G. H. (1995). Electrophysiology of Mind: Event-related Brain Potentials and Cognition. New York: Oxford University Press.

Sabourin, L., \& Stowe, L. A. (2008). Second language processing: when are first and second languages processed similarly? Second Language Research, 24(3), 397-430. https://doi.org/10.1177/0267658308090186

Schütze, C. (1996). The Empirical Base of Linguistics: Grammaticality Judgments and Linguistic Methodology. Chicago: Chicago University Press.

Wasow, T., \& Arnold, J. (2005). Intuitions in linguistic argumentation. Lingua, 115(11), 1481-1496. https://doi.org/10.1016/j.lingua.2004.07.001

White, L. (1989). Universal Grammar and Second Language Acquisition. Amsterdam: John Benjamins.

White, L. (2003). Second Language Acquisition and Universal Grammar. Cambridge: Cambridge University Press. https://doi.org/10.1017/CBO9780511815065

\section{Copyrights}

Copyright for this article is retained by the author(s), with first publication rights granted to the journal.

This is an open-access article distributed under the terms and conditions of the Creative Commons Attribution license (http://creativecommons.org/licenses/by/4.0/). 